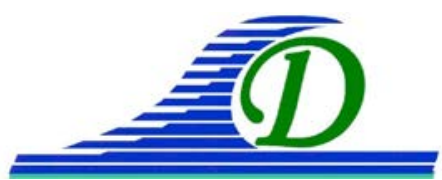
XIII ${ }^{\text {èmes }}$ Journées Nationales Génie Côtier - Génie Civil
Dunkerque, 2-4 juillet 2014

DOI:10.5150/jngcgc.2014.065 C Editions Paralia CFL

disponible en ligne - http://www.paralia.fr - available online

\title{
Suivi géoélectrique des intrusions d'eau salée sous un cordon dunaire côtier au cours d'une marée
}

\author{
Clara JODRY ${ }^{1}$, Sérgio PALMA LOPES ${ }^{2}$, Anaëlle JOUBERT ${ }^{2}$, \\ Martin SANCHEZ ${ }^{3}$, Philippe CÔTE ${ }^{4}$
}

1. Université de Nantes, UMS 3281 OSUNA, UMR 6112 CNRS - LPG,

IFSTTAR, dépt GERS, laboratoire Auscultation et Imagerie (AI),

Route de Bouaye CS4, point courrier 87, 44344 Bouguenais Cedex, France. clara.jodry@ifsttar.fr

2. LUNAM, IFSTTAR, dépt GERS, laboratoire Auscultation et Imagerie (AI), Route de Bouaye CS4, point courrier 87, 44344 Bouguenais Cedex, France. sergio.lopes@ifsttar.fr,anaelle.joubert@ifsttar.fr

3. Université de Nantes, UFR Sciences et Techniques, UMR 6112 CNRS - LPG, 2 rue de la Houssinière, BP 92208, 44322 Nantes, France.

martin.sanchez@univ-nantes.fr

4. LUNAM, IFSTTAR, dépt GERS

Route de Bouaye CS4, point courrier 87, 44344 Bouguenais Cedex, France.

philippe.cote@ifsttar.fr

\section{Résumé :}

Dans le cadre d'un projet de recherche visant à développer une méthodologie géophysique pour le suivi temporel des échanges eau de mer / eau douce sous les dunes côtières, un suivi expérimental par tomographie de résistivité électrique a été mené. Des mesures géoélectriques ont été acquises au cours d'une marée sur l'île de Noirmoutier. Les données brutes présentent du bruit instrumental et environnemental, ainsi que des anomalies dues à des objets métalliques en place. Malgré ces perturbations, l'effet du mouvement de l'eau salée dans le sous-sol, qui se traduit par des variations de résistivité dans le temps, est détectable sur une des lignes de mesure. Nous avons utilisé une méthode de normalisation qui permet une meilleure mise en évidence des faibles variations mesurées, dans un milieu fortement contrasté et en présence de bruit. Les images de résistivités normalisées inversées, qui expriment des variations par rapport à un temps de référence, montrent des évolutions faibles mais significatives. Ces premières conclusions seront validées par la pose d'instrumentation et la réalisation de sondages. Cette expérimentation contribue à nos développements méthodologiques, tant au niveau du protocole d'acquisition que de la méthode d'inversion.

Mots-clés : Tomographie de résistivité électrique, Dune côtière, Hydrogéologie, Cycle de marée, Suivi temporel, Bruit. 


\section{Introduction}

Le cordon dunaire est un système sédimentaire dynamique formé par des dépôts de sable répartis sur un linéaire parallèle à la côte. En France, 45\% des côtes métropolitaines sont potentiellement concernés par ce phénomène de façon plus ou moins marquée (COLLECTIF, 2013). Ces crêtes naturelles ont souvent des altitudes supérieures à la hauteur moyenne de la mer, ce qui leur permet d'amortir les agressions marines. De nombreuses études, regroupant plusieurs domaines des géosciences, portent sur les différents forçages (vents, mer, tourisme et aménagement du territoire) dans le but de retracer la dynamique de formation et d'érosion des dunes côtières et ainsi prédire leur évolution (CLEMMENSEN et al., 2013). Toutefois, on s’intéresse ici aux processus hydrogéologiques ayant lieu sous la dune de sable, et reliant les intrusions marines aux écoulements d'eau de pluie infiltrée. Pour cela on utilise une méthode géophysique non intrusive, la tomographie de résistivité électrique (TRE). En effet le résistivité de l'eau de mer est de l'ordre de 0.2 à $1 \Omega \mathrm{m}$ tandis qu'elle est de 5 à $100 \Omega \mathrm{m}$ pour l'eau douce (PALACKY, 1991). Ainsi, l'intrusion marine induit un panache de faibles résistivités dans un environnement de plus fortes résistivités. Notons que les techniques de résistivité électrique se sont déjà montrées efficaces pour détecter et suivre une intrusion d'eau salée (e.g. DE FRANCO et al., 2009 ; MORROW et al., 2010 ; POULSEN et al., 2010 ; CHITEA et al., 2011 ; CHOUTEAU et al., 2012). Ces études montrent également la sensibilité des caractéristiques de la zone d'intrusion à différents paramètres environnementaux (pluviométrie, coefficient de marée, hauteur de la houle) ainsi qu’à la structure géologique. L’intrusion marine, et par conséquent son impact sur des mesures géoélectriques, sont très différents selon le site et l'échelle de temps et d'espace considérés.

Dans le cadre d'un projet de recherche régional (RS2E-OSUNA), notre objectif sur le long terme est de développer une méthodologie géophysique permettant le suivi temporel des changements d'état hydrique du sol sous les dunes côtières. Une première expérience de suivi temporel, sur un cycle de marée et considérant les vingt premiers mètres de profondeur, a été menée sur l'île de Noirmoutier. A ce stade du projet, le dispositif de TRE devait permettre de caractériser l'intrusion d'eau salée : distribution spatiale, variations temporelles et déphasage par rapport au cycle de marée. L’objectif de cet article est de présenter les résultats de cette expérience.

Après une présentation du site et des mesures réalisées, nous décrivons le filtrage appliqué aux données brutes affectées par plusieurs sources de bruit. Ensuite, nous présentons les premiers résultats d'inversion. Les variations temporelles que l'on cherche à suivre semblent avoir des amplitudes très faibles. Nous terminons par une discussion ouvrant sur des perspectives. 


\section{XIII $I^{\text {èmes }}$ Journées Nationales Génie Côtier - Génie Civil \\ Dunkerque, 2-4 juillet 2014}

\section{L’expérimentation}

\subsection{Le site atelier}

Situé sur la côte ouest de l'île de Noirmoutier, le site expérimental est une portion de la dune de l'Hommée correspondant à $100 \mathrm{~m}$ de front dunaire (5 m de hauteur, $70 \mathrm{~m}$ de largeur). Il est exposé à une forte dynamique éolienne et maritime venue de l'Océan Atlantique.

La formation du cordon sableux a été favorisée par des bancs de calcaires gréseux que l'on retrouve théoriquement à $-4 \mathrm{~m}$ d'altitude IGN-NGF69. Épais d'une dizaine de mètres, ils constituent un aquifère contenant une nappe d'eau salée alimentée par l'océan. Une couche d'argile sableuse sépare cet aquifère des sables dunaires et la nappe libre qu'ils contiennent (FAISSOLLE \& ROY, 1994).

Une volonté récente de conserver la dynamique naturelle des dunes côtières a conduit à un contrôle souple (FAVENNEC, 2002). Entre autres techniques, des ganivelles (brisevents comprenant des fils métalliques) et de la végétation sont installés de façon systématique.

\subsection{Acquisitions de données géoélectriques}

L'expérimentation a consisté à effectuer des acquisitions de TRE sur un demi-cycle de marée afin de suivre les mouvements de l'intrusion marine et sa répartition spatiale. Le dispositif utilisé comprend deux lignes perpendiculaires d'électrodes définissant ainsi un profil transversal suivant la topographie de la dune de la terre vers la mer et un profil longitudinal en pied de dune côté plage (voir figure 1a et 1b). L'intervention a eu lieu sur une période de 9 heures, avec une acquisition toutes les heures pour chaque profil (voir figure 1c). Le profil transversal a une longueur totale de $117.5 \mathrm{~m}$ contre $94 \mathrm{~m}$ pour le profil longitudinal, incluant respectivement 45 électrodes espacées de $2.5 \mathrm{~m}$ et 48 électrodes espacées de $2 \mathrm{~m}$. La séquence d'acquisition utilisée est de type "WennerSchlumberger" (MARESCOT, 2006) basée sur des quadripôles A-M-N-B avec les électrodes de mesure $\mathrm{M}$ et $\mathrm{N}$ situées entre les électrodes d'injection $\mathrm{A}$ et $\mathrm{B}$. Notons "a" la distance $M N$ et " $n * a$ " la distance $A M=N B$, avec "n" nombre entier compris entre 1 et 8. Chacune des lignes d'électrodes permet ainsi d'obtenir entre 871 et 987 mesures de résistivité apparente. Une résistivité apparente est une mesure “intégrante”, sensible aux propriétés électriques du milieu dans un certain volume d'investigation (qui dépend de l'écartement entre électrodes). Pour estimer la distribution de résistivité "vraie" du terrain dans une coupe verticale il est nécessaire d'inverser ces données. Pour cela on utilise le logiciel commercial RES2Dinv (LOKE \& BARKER, 1996) qui nous permet de choisir plusieurs paramètres d'inversion en fonction de l'a priori sur les mesures et sur le terrain (algorithme de minimisation aux moindres carrés amortis). 

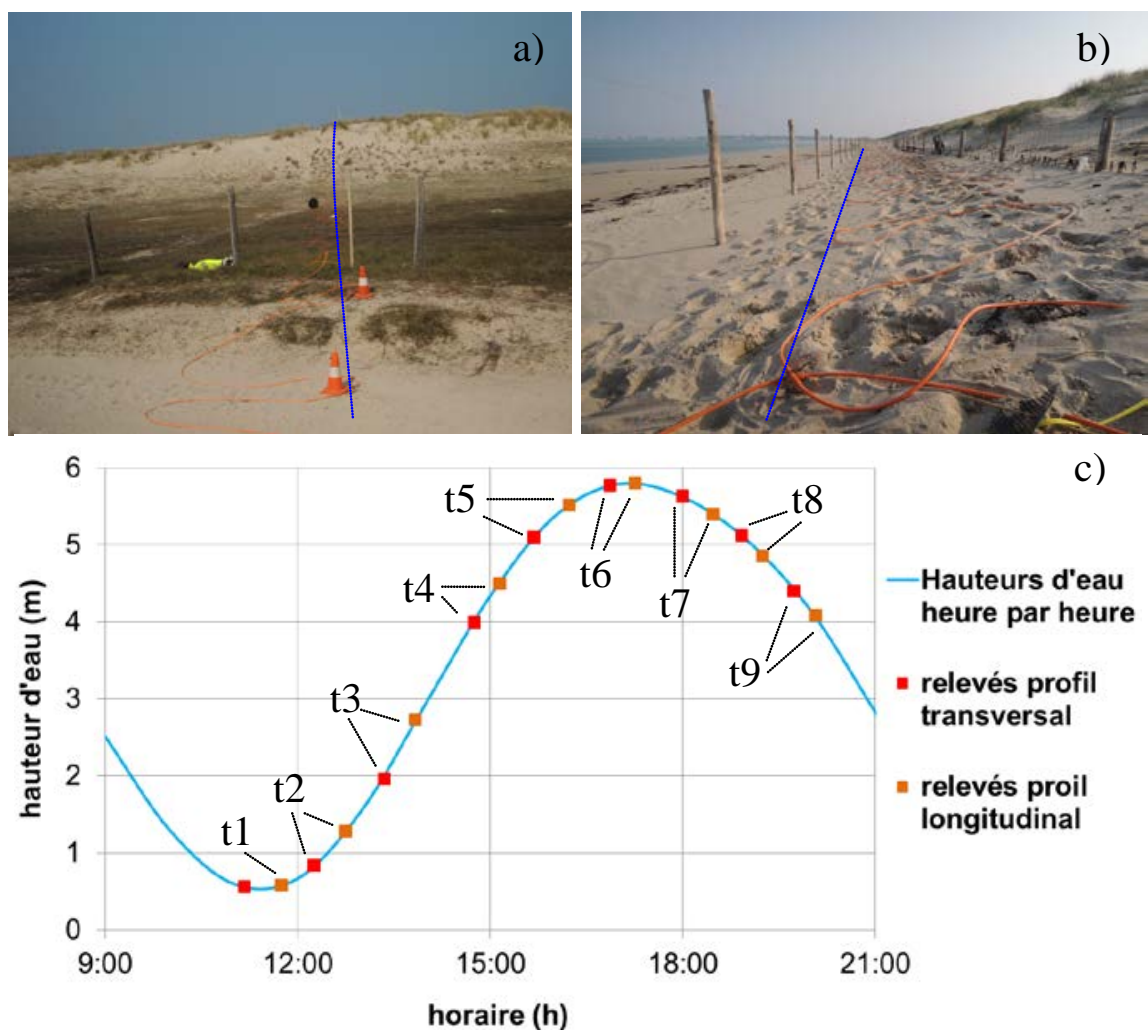

c)

-Hauteurs d'eau heure par heure

- relevés profi

transversal

relevés proil longitudinal

Figure 1. a) Vue du profil de TRE transversal à la dune tracé en bleu., b) Vue du profil longitudinal de TRE tracé en bleu, et c) courbe temporelle de la marée du 28 mars 2013 avec des instants $t 1$ à $t 9$ des acquisitions de TRE (O SHOM).

\section{Analyse}

\subsection{Diagnostic et prétraitement des mesures}

Les résistances de contact électrique entre les électrodes et le sol diminuent au cours du temps à partir de l'installation des électrodes, phénomène déjà constaté par d'autres auteurs (LABRECQUE et al., 1996). Ainsi les résultats présentés dans la suite concernent uniquement les temps $\mathrm{t} 4 \mathrm{à} \mathrm{t} 9$, dont les résistances de contact restent comprises entre 2 et $12 \mathrm{k} \Omega$ (des résistances de prise inférieures à $20 \mathrm{k} \Omega$ sont généralement jugées très favorables à la qualité des mesures). Chaque réponse électrique est moyennée sur plusieurs mesures répétées. L'analyse de l'écart type de chaque réponse électrique (non présentée ici) indique un bruit aléatoire lié aux conditions du site, d'amplitude variable, supérieure pour le profil transversal et qui décroit au cours du temps. Enfin la présence d'un bruit systématique a été mise en évidence. Il s'agit d'oscillations spatiales de forte amplitude et reproductibles (voir figure 2a). Ce bruit, qui impacte un nombre significatif de mesures, s'avère être spatialement corrélé avec la présence, constatée ou supposée, des ganivelles ensablées 


\section{XIII $I^{\text {èmes }}$ Journées Nationales Génie Côtier - Génie Civil \\ Dunkerque, 2-4 juillet 2014}

dans la dune. Nous supposerons ici que ce bruit systématique perturbe peu une analyse des évolutions temporelles.

Ainsi, ont été supprimées, les données de faible rapport signal sur bruit (trop grands espacements entre électrodes), celles d'écart-type supérieur à $5 \%$, et enfin, nous avons supprimé les résistivités apparentes inférieures à $0.5 \Omega \mathrm{m}$ (valeur minimale possible pour un sable de plage saturé en eau de mer, PALACKY, 1991). Au total, ces filtres réduisent le nombre de données de 33\% en moyenne, permettant ainsi de ne conserver que les mesures les plus fiables pour analyser les variations temporelles recherchées.

\subsection{Variation temporelle des mesures}

Les variations temporelles des résistivités apparentes ont été étudiées en lien avec le phénomène de marée. Les résultats du profil longitudinal sont montrés figure $2 b$, où l'on peut constater l'existence de variations globales (sur l'ensemble du profil) que nous supposons dues à l'évolution du milieu.
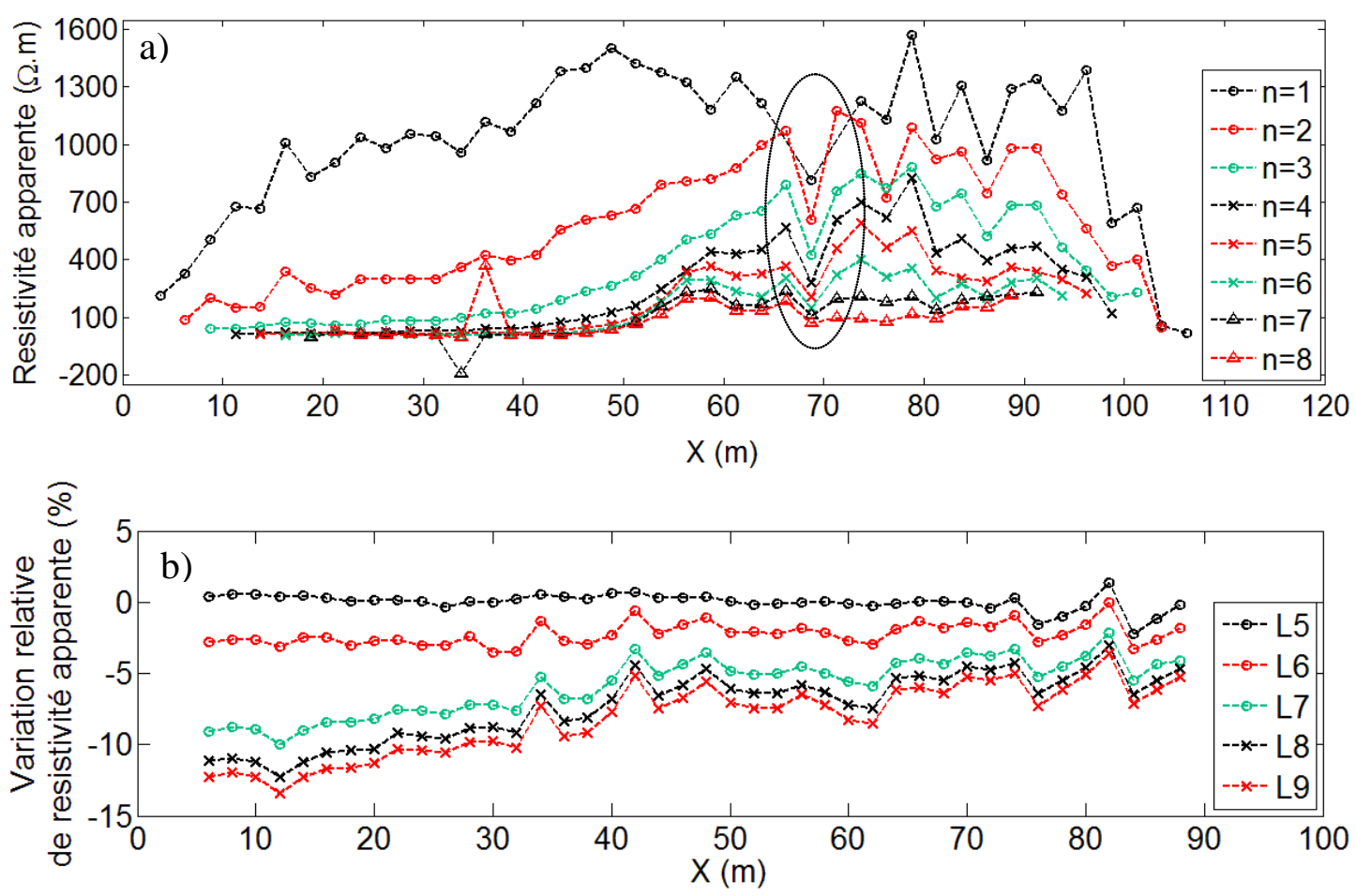

Figure 2. a) Résistivités apparentes mesurées le long du profil transversal au temps t4 pour $a=2.5 \mathrm{~m}$ (bruit systématique encerclé) et b) variations relatives de résistivité apparente le long du profil longitudinal par rapport au temps t4 pour $n=1$ et $a=4 \mathrm{~m}$.

Ces changements significatifs sur la ligne longitudinale peuvent atteindre $13 \%$ sur la seconde moitié du cycle de marée. Sur la ligne transversale, les variations relatives des 
résistivités apparentes (non présentées ici) sont bruitées et peu significatives, indiquant une adaptation insuffisante de notre protocole d'acquisition aux échelles du phénomène.

\subsection{Modèle géoélectrique de la dune}

Avant d'étudier les variations d'état du milieu au cours de la marée, il est important d'obtenir la distribution spatiale de la résistivité électrique dans ce milieu (à un instant donné) et de faire la correspondance avec les couches géologiques attendues. La figure 3 présente un résultat typique, avec de la gauche vers la droite, la dune grise (faible altitude et faible couverture de sable sec), la dune blanche (topographie marquée et importante épaisseur de sable sec électriquement résistant), et le début de la plage à peine visible (diminution de la résistivité en surface). Ce résultat a été obtenu après réglage des paramètres d'inversion pour tenir compte des informations a priori : fort contraste de résistivité entre la dune blanche et les milieux sous nappe, d'eau douce à salée, ainsi qu'un milieu relativement tabulaire sous la dune, et présence de bruit et de perturbations (effet des ganivelles). Cette coupe, bien que globalement cohérente avec des résultats antérieurs (BUANNIC, 2012), devra être confirmée par des sondages mécaniques.

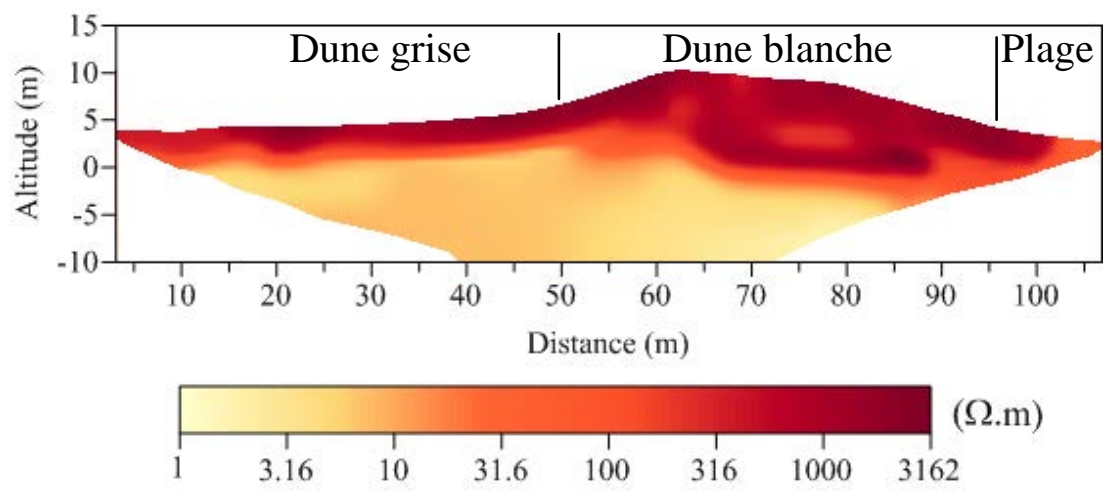

Figure 3. Coupe de résistivité du profil transversal à la dune obtenue par inversion après filtrage des données enregistrées au temps 4 à l'itération 5.

\subsection{Inversion des variations temporelles}

Notre objectif étant de mettre en évidence les variations temporelles du modèle géoélectrique de la dune, nous avons procédé à des séries de tests d'inversion des variations des données mesurées. Plusieurs stratégies et algorithmes sont régulièrement discutés dans la littérature (HAYLEY et al., 2011). Les approches les plus employées (inversion conjointe de toutes les séries temporelles avec différentes formes de contraintes entre ces séries), et avec le logiciel RES2Dinv, n’ont pas permis de visualiser des évolutions significatives et cohérentes, ce que nous attribuons aux différentes sources de bruit et à la faible amplitude des variations de résistivités apparentes mesurées, dans un milieu très fortement contrasté (voir figure 3). 


\section{XIII $I^{\text {èmes }}$ Journées Nationales Génie Côtier - Génie Civil \\ Dunkerque, 2-4 juillet 2014}

Nous avons donc tenté une autre approche, suggérée par DAILY et al. (2004), qui consiste à inverser des données normalisées afin de mettre en valeur les faibles amplitudes de variations et qui est dite efficace en présence d'un milieu 3D fortement contrasté :

$\rho_{a}^{n}=\frac{\rho_{a}^{t}}{\rho_{a}^{t 4}} \rho_{a}^{h}$

où : $\rho_{a}{ }^{n}$ est la résistivité apparente normalisée, $\rho_{a}{ }^{t}$ est la résistivité apparente mesurée au temps considéré, $\rho_{a}{ }^{t 4}$ la résistivité apparente mesurée au temps de référence 4 et $\rho_{a}{ }^{h}$ une résistivité apparente que l'on mesurerait si le milieu était homogène de résistivité $100 \Omega \mathrm{m}$ (valeur arbitraire).

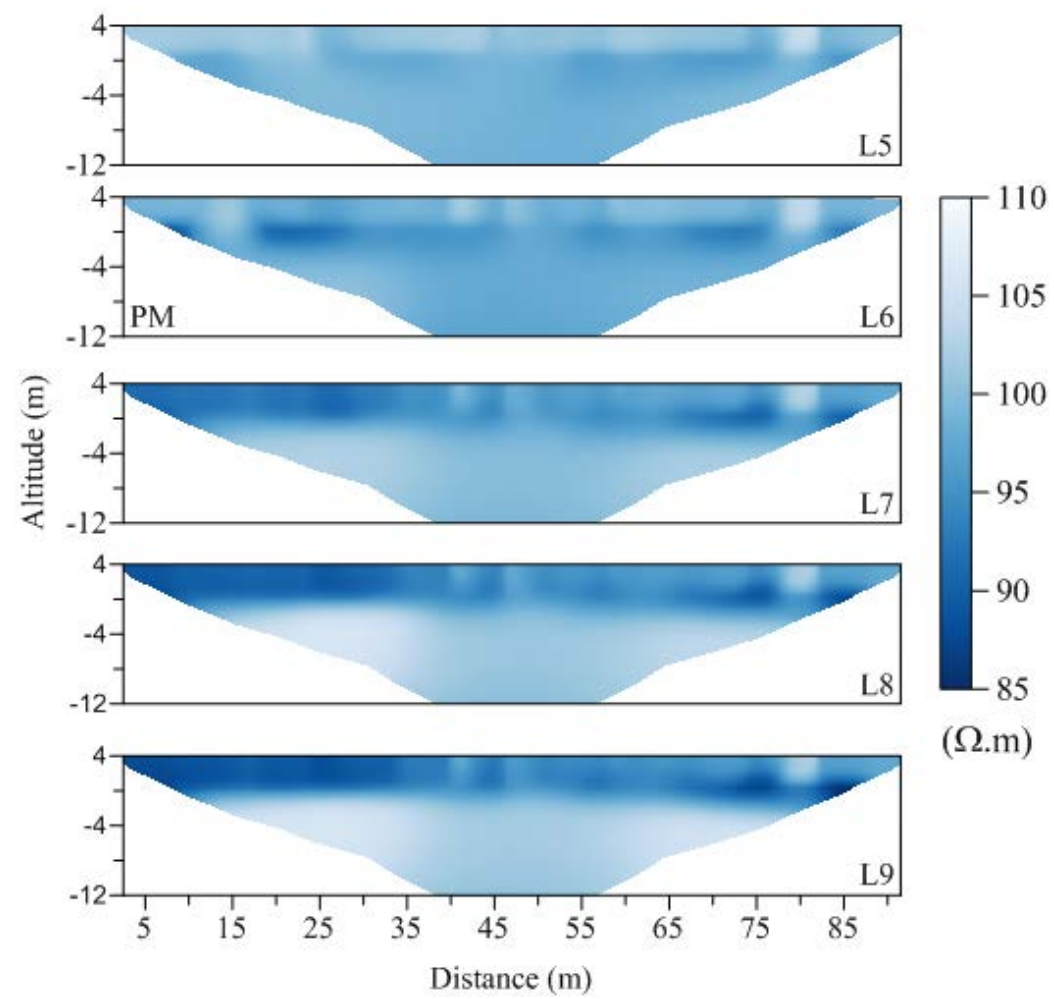

Figure 4. Coupes de variations de résistivité du profil longitudinal, inversées avec des données normalisées au temps de référence 4 (PM : niveau de pleine mer).

On obtient alors une image inversée qui illustre des variations entre le temps 4 de référence et le temps étudié, dans laquelle les valeurs supérieures à $100 \Omega \mathrm{m}$ indiquent une augmentation de résistivité et inversement. Nous ne présentons le résultat de cette approche que sur le profil longitudinal, qui présente des variations plus significatives des signaux enregistrés.

On peut voir une diminution progressive de la résistivité atteignant environ $15 \%$ en moyenne sur la durée du demi-cycle considéré. Ces variations sont spatialement 
localisées dans ce qui pourrait être l'aquifère sableux au-dessus de la couche d'argile (théoriquement au-dessus de $0 \mathrm{~m}$ d'altitude IGN-NGF69), zone supposée de changement de salinité de l'eau avec l'intrusion saline. Les calcaires gréseux montrent quant à eux une augmentation de la résistivité ne dépassant pas $10 \%$. On note cependant que ces résultats peuvent être affectés par l'influence de la mer sur les mesures électriques : la mer, qui représente une masse conductrice latérale par rapport à notre ligne longitudinale, s'éloigne au cours du temps et peut induire des augmentations de résistivité vers le bas des coupes de variations dus à ces effets 3D dans une inversion 2D. Ces interprétations sont donc à approfondir, et surtout à confirmer par des sondages et un suivi piézométrique approprié.

\section{Perspectives à court terme}

Nous devons d'abord confirmer que notre protocole de suivi couvre des échelles de temps et d'espace adaptées à la dynamique de ce site : de nouveaux essais d'acquisition sont en cours afin de tester plusieurs approches de suivi. La prochaine mise en place de piézomètres sur la dune grise parallèlement au profil transversal avec mesure continue des niveaux de nappe, de la conductivité électrique et de la température, permettra de quantifier ces phénomènes à surveiller. À cette occasion, des sondages mécaniques et des prélèvements sont prévus. Ils permettront de préciser la nature et l'épaisseur des couches géologiques investiguées. On utilisera ensuite un code d'imagerie de résistivité plus adéquat (FARGIER, 2011), avec la prise en compte de ces informations géologiques dans un modèle 3D. Ce code sera adapté à la problématique du site, en intégrant un algorithme d'inversion des variations temporelles qui sera choisi pour sa capacité à mettre en évidence des variations faibles en présence de forts contrastes et de bruit. Parallèlement, on améliorera les prétraitements des données brutes pour diminuer l'influence du bruit ambiant et des structures anthropiques (ganivelles). Enfin, un modèle réduit va être réalisé afin d'étudier l'influence des ganivelles sur des mesures de TRE en fonction de paramètres géométriques et physiques.

\section{Conclusions}

Un suivi géoélectrique d'une dune côtière a été réalisé sur un demi-cycle de marée. Les résultats mettent en évidence les difficultés à traiter, inverser puis interpréter des signaux dont les variations sont de faible amplitude et qui, même s’ils dévoilent une certaine évolution temporelle, restent très perturbés par les sources de bruit environnantes. L'interprétation simple proposée en fonction de la marée reste à valider par des observations directes. Cette analyse ouvre ainsi des pistes de recherches: optimiser le protocole d'acquisition aux échelles temporelle et spatiale réelles de l'intrusion marine, et développer la modélisation et l'inversion pour les adapter aux spécificités du milieu investigué pour atteindre les objectifs méthodologiques du projet sur le long terme. 


\section{XIII $I^{\text {èmes }}$ Journées Nationales Génie Côtier - Génie Civil \\ Dunkerque, 2-4 juillet 2014}

\section{Remerciements}

La dune de l'Hommée est gérée par l’Office National des Forêts qui a autorisé nos interventions, grandement facilitées aussi par l'aide gracieuse de la Communauté de communes de l'île de Noirmoutier.

Les auteurs remercient les deux relecteurs anonymes qui ont permis d'améliorer significativement cet article.

\section{Références bibliographiques}

BUANNIC E. (2012). Surveillance des protections fluviales et littorales par méthodes géoélectriques. Rapport de stage de master 2, Université de Grenoble, pp 1-72

COLLECTIF (2013). Le littoral. Géosciences, la revue du BRGM pour une Terre durable, Vol. 17. http://editions.brgm.fr/dcenewsFile?ID=1803

CHITEA F., GEORGESCU P., IOANE D. (2011). Geophysical detection of marine intrusions in Black Sea coastal areas (Romania) using VES and ERT data. In Geo-Eco Marina, pp 95-102.

CHOUTEAU M.C., LABIAU M., TAVIAU C., CHAPUIS R., CHOU C.T.K. (2012). ERT Monitoring of tide-induced water flow in a shallow beach aquifer. 18th European Meeting of environmental and Engineering Geophysics.

CLEMMENSEN L.B., BENDIXEN M., HEDE M.U., KROON A., NIELSEN L., MURRAY A.S. (2013). Morphological records of storm floods exemplified by the impact of the 1872 Baltic storm on a sandy spit system in south-eastern Denmark. Earth Surface Processes and Landforms. http://dx.doi.org/10.1002/esp.3466

DAILY W.I., RAMIREZ A.B., BINLEY A., LABRECQUE D. (2004). Electrical resistance tomography. The Leading Edge, Vol. 2, pp 438-442. http://dx.doi.org/10.1190/1.1729225

DE FRANCO R., BIELLA G., TOSI L., TEATINI P., LOZEJ A., CHIOZZOTTO B., GIADA M. RIZZETTO F. CLAUDE C. MAYER A. BASSAN V., GASPARETTOSTORI G. (2009). Monitoring the saltwater intrusion by time lapse electrical resistivity tomography: The Chioggia test site (Venice Lagoon, Italy). Journal of Applied Geophysics, Vol. 69(3-4), pp 117-130. http://dx.doi.org/10.1016/j.jappgeo.2009.08.004

FAISSOLLE F., ROY C. (1994). Les eaux souterraines de l'ile de noirmoutier, caractéristiques, utilisation, gestion. Vendée Conseil Général, n 94.176, pp 1-12.

FARGIER Y. (2011). Développement de l'imagerie de résistivité électrique pour la reconnaissance et la surveillance des ouvrages hydrauliques en terre. Thèse Université de Nantes, $244 \mathrm{p}$.

FAVENNEC J. (2002). Contrôle souple et réhabilitation des dunes littorales non boisées de la côte atlantique. Connaissance et gestion durable des dunes de la côte atlantique. Les dossiers forestiers ONF n²11, pp 205-223. 
Thème 3 - Instrumentation, mesures, imagerie et télédétection

HAYLEY K., PIDLISECKY A., BENTLEY L.R. (2011). Simultaneous time-lapse electrical resistivity inversion. Journal of Applied Geophysics, Vol. 75(2), pp 401-411. http://dx.doi.org/10.1016/j.jappgeo.2011.06.035

LABRECQUE D.J., MILETTO M., DAILY W., RAMIREZ A., OWEN E. (1996). The effect of noise on Occam's inversion of resistivity tomography data. Geophysics, Vol. 61(2), pp 538-548.

LOKE M.H., BARKER R.D. (1996). Rapid least-squares inversion of apparent resistivity pseudosections by a quasi-Newton method. Geophysical Prospecting, Vol. 44, pp 131-152. http://dx.doi.org/10.1111/j.1365-2478.1996.tb00142.x

MARESCOT L. (2006). Introduction à l'imagerie électrique du sous-sol. Bulletin de La Société Vaudoise des Sciences Naturelles, Vol. 90(1), pp 23-40.

MORROW F. J., INGHAM M. R., McCONCHIE J. A. (2010). Monitoring of tidal influences on the saline interface using resistivity traversing and cross-borehole resistivity tomography. Journal of Hydrology, Vol. 389(1-2), pp 69-77. doi:10.1016/.j.hydrol.2010.05.022

PALACKY G.J. (1991). Resistivity characteristics of geological targets. Dans M.N. Nabighian (ED.), Electromagnetic Methods in Applied Geophysics, Soc. Explor. Geophys., Vol. 1, pp 53-129.

POULSEN S.E., RASMUSSEN K. R., CHRISTENSEN N.B. (2010). Evaluating the salinity distribution of a shallow coastal aquifer by vertical multielectrode profiling (Denmark). Hydrogeology Journal, Vol. 18(1), pp 161-171. 